\title{
The influence of local pressure on evaluation parameters of skin blood perfusion and fluorescence
}

E. A. Zherebtsov, K. Y. Kandurova, E. S. Seryogina, I. O. Kozlov, V. V. Dremin, et al.

E. A. Zherebtsov, K. Y. Kandurova, E. S. Seryogina, I. O. Kozlov, V. V. Dremin, A. I. Zherebtsova, A. V. Dunaev, I. Meglinski, "The influence of local pressure on evaluation parameters of skin blood perfusion and fluorescence," Proc. SPIE 10336, Saratov Fall Meeting 2016: Optical Technologies in Biophysics and Medicine XVIII, 1033608 (24 March 2017); doi: 10.1117/12.2267957

SPIE Event: Saratov Fall Meeting 2016: Fourth International Symposium on Optics and Biophotonics, 2016, Saratov, Russian Federation 


\title{
The influence of local pressure on evaluation parameters of skin blood perfusion and fluorescence
}

\author{
E.A. Zherebtsov ${ }^{\text {a,b, }}$, K.Y. Kandurova ${ }^{a}$, E.S. Seryogina ${ }^{a}$, I.O. Kozlov ${ }^{a}$, V.V. Dremin ${ }^{\text {a }}$, \\ A.I. Zherebtsova ${ }^{a}$, A.V. Dunaev ${ }^{a}$, I. Meglinski ${ }^{b}$ \\ ${ }^{a}$ Biomedical Photonics Instrumentation Group, Scientific-Educational Centre of "Biomedical \\ Engineering", Orel State University named after I.S. Turgenev, Komsomolskaya St. 95, \\ Orel, Russia, 302026 \\ ${ }^{b}$ University of Oulu, Faculty of Information Technology and Electrical Engineering, \\ Opto-Electronics and Measurement Techniques, Erkki Koiso-Kanttilankatu 3, Oulu, Finland, 90570
}

\begin{abstract}
This article presents the results of the study of the pressure applied on optical diagnostic probes as a significant factor affecting the results of measurements. During stepwise increasing and decreasing of local pressure on skin we conducted measurements using the methods of laser Doppler flowmetry and fluorescence spectroscopy. It was found out that pressure on optical probe has sufficient impact on skin microcirculation to affect registered fluorescence intensity. Data obtained in this study are of interest for design and development of diagnostic technologies for wearable devices. This data will also inform further investigation into issues of compensation of blood absorption influence on fluorescence spectrum, allowing increased accuracy and reproducibility of measurements by fluorescence spectroscopy methods in optical diagnosis.
\end{abstract}

Keywords: wearable technology, local pressure, non-invasive diagnostics, laser Doppler flowmetry, blood microcirculation, fluorescence spectroscopy.

\section{INTRODUCTION}

It is well known that the pressure applied on optical diagnostic probes is a significant factor affecting the results of measurements. One of the main causes of such effect is the influence of the pressure on local blood flow ${ }^{1-3}$. Taking into account holding pressure is necessary when developing new wearable electronics. Therefore, investigating the influence of local pressure on measurement results of wearable devices for optical diagnostic technologies is a relevant issue at present.

In the recent years fluorescence spectroscopy (FS) and laser Doppler flowmetry (LDF) have been successfully used in studies of biological objects at the tissue level, which allows the monitoring of temporal and spatial dynamics of the microcirculatory-tissue systems $\mathrm{s}^{4-7}$. FS method is based on analyzing the characteristics of induced endogenous fluorescence in probed biological tissues with low-intensity optical radiation at certain wavelengths ${ }^{8,9}$. However, this method has a weak methodological support for use in clinical practice ${ }^{10,11}$, because recorded spectra are influenced by many factors. One of the factors is the alteration of blood flow in the biological tissue.

One of the methods which allows to assess the blood flow in covering tissues is the method of LDF. This method is based on the detection of the Doppler shift of laser radiation produced by reflection from moving red blood cells in the microvasculature ${ }^{12}$. The result of LDF measurements represents "index of blood microcirculation $\left(\mathrm{I}_{\mathrm{m}}\right)$ " or "perfusion", assessed in conventional perfusion units (PU). Temporal records of the parameter reveal a complex, non-periodic process of the blood microcirculation regulation in skin and mucosa. With LDF it is possible not only to non-invasively evaluate the intensity of the blood flow in the microcirculation bed of the blood flux, but also to discover and explore the collective rhythmic processes of microcirculation ${ }^{12}$.

*Correspondence: zherebzow@gmail.com; phone+7 953 6151192; www.bmecenter.ru/en/vascular-control 
Pressure is a well-known technique for controlling optical properties of biological tissue. It increases diagnostic depth and volume as well as allowing evaluation of actual values of optical parameters of biological tissue in vivo (parameters of absorption, scattering, polarization, fluorescence ${ }^{13}$, etc.) by reducing the influence of blood absorption. The research aim of this work is the experimental study of the influence of local pressure on the skin - by optical probe - on measurement results from Doppler flowmetry (LDF) and fluorescence spectroscopy (FS).

\section{THE METHOD OF RESEARCH}

Experiments were conducted using the optical non-invasive diagnostic device "LAKK-M" (SPE "LAZMA" Ltd, Russia) (Fig.1a). This device has embedded LDF (1064 nm) and fluorescence spectroscopy (excitation at wavelengths 365, 450, 532 and $637 \mathrm{~nm}$ ) diagnostic channels. To change the pressure value fixed on the optical probe, special tooling has been developed and manufactured using a 3D-printer (Fig. 1b).

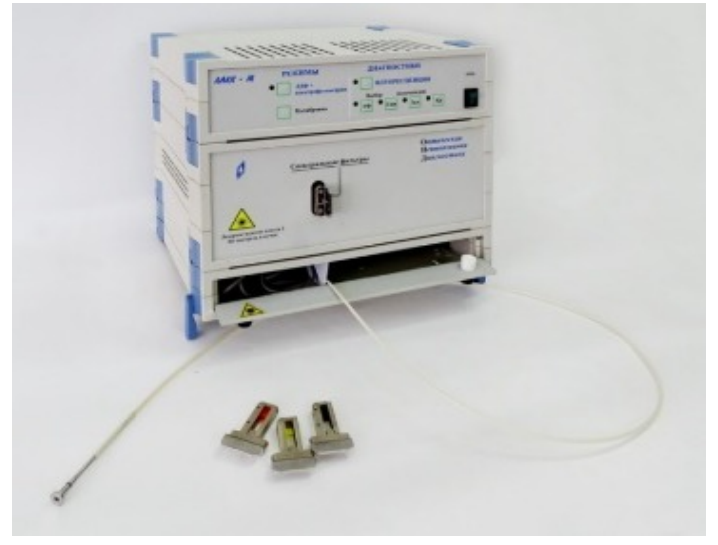

(a)

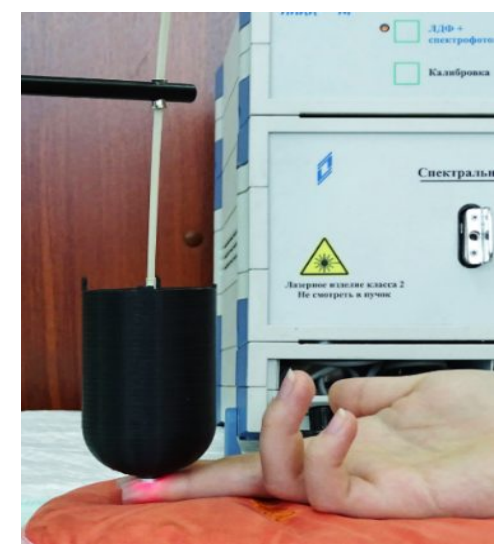

(b)

Figure 1. Experimental equipment: "LAKK-M" device (a) and Placement of the special tooling for optical fiber of "LAKK-M" device on the finger (b).

A total of 7 healthy volunteers aged $24 \pm 7$ years were engaged in the research. Each experiment lasted about 50 minutes. At the first stage, influence of pressure on averaged LDF-signal level was investigated, alongside characteristics of Doppler spectra. At the second stage, the influence of pressure on registered intensity of skin fluorescence was investigated. Before the main experiment background fluorescence at four excitation wavelengths was recorded from skin surface measurement area. Finally, fluorescence spectra measurements were performed with stepwise increasing and decreasing pressure on optical probe.

During each experiment, pressure was changed stepwise from 0 to $40 \mathrm{kPa}$ and after that it was reduced back to $0 \mathrm{kPa}$. Palmar surface of the middle finger of right hand was selected as area of interest due to frequent use of this area for optical non-invasive measurements.

\section{RESULTS AND DISCUSSION}

Example of obtained perfusion distribution by frequency of Doppler shift during the one of experiments is present in Fig.2. The processing of Doppler spectra allowed the identification of the effect of velocity re-distribution of moving $\mathrm{RBC}$ in diagnostic volume when the pressure was changed. This figure clearly shows the moment of perfusion increasing in the low frequency range with increasing pressure. In general case the low frequency correlate with low speed of RBC in the sampling volume of the probe. So, there is velocity redistribution of the RBC during alternation of the external pressure on the optical probe. Furthermore, at certain moment (for this case at pressure approximately $15 \mathrm{kPa}$ ) blood flow goes down (and registers at the noise level). After this moment, the processes in the sampling volume of the probe 
correspond to local occlusion with potential hypoxia of the tissues. Meanwhile, the pressure of occlusion onset depends on the individual features of the volunteer.

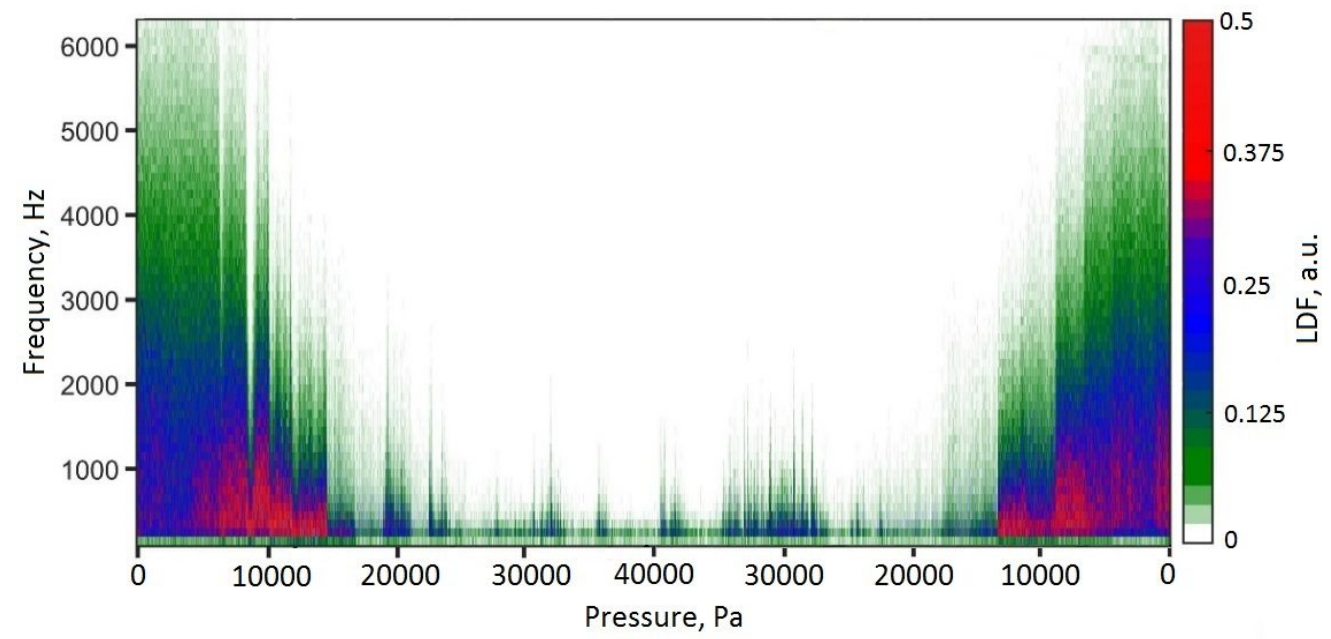

Figure 2. Visualization of perfusion distribution by frequency of Doppler shift during local pressure test.

Obtained experimental data were used to generate an averaged curve of blood perfusion reduction and to represent it in exponential approximation (Fig. 3). On average, procedure using maximum applied pressure led to a fall in perfusion level by $85 \%$ from the initial level. Even at pressures of $5 \mathrm{kPa}$ the perfusion level decreased by $25 \%$.

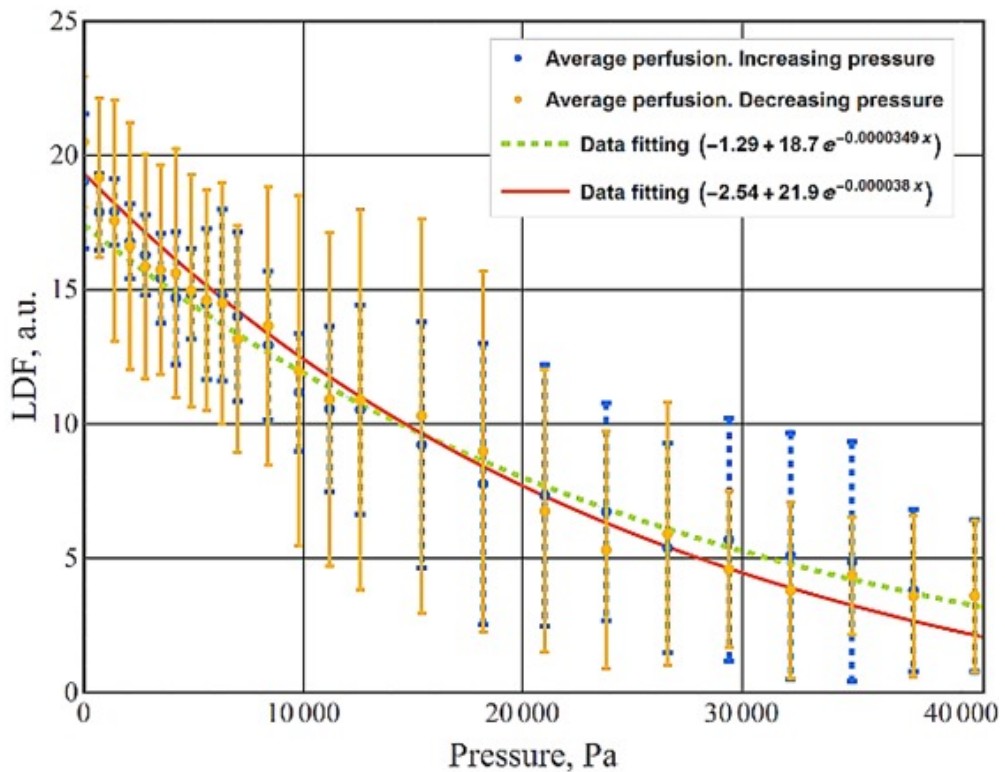

Figure 3. Average index of microcirculation (perfusion) for all volunteers

In Fig. 4 typical fluorescence spectra at pressures $0 \mathrm{kPa}, 5 \mathrm{kPa}$ and $40 \mathrm{kPa}$ for wavelengths $365 \mathrm{~nm}$ (a) $450 \mathrm{~nm}$ (b) are presented. 


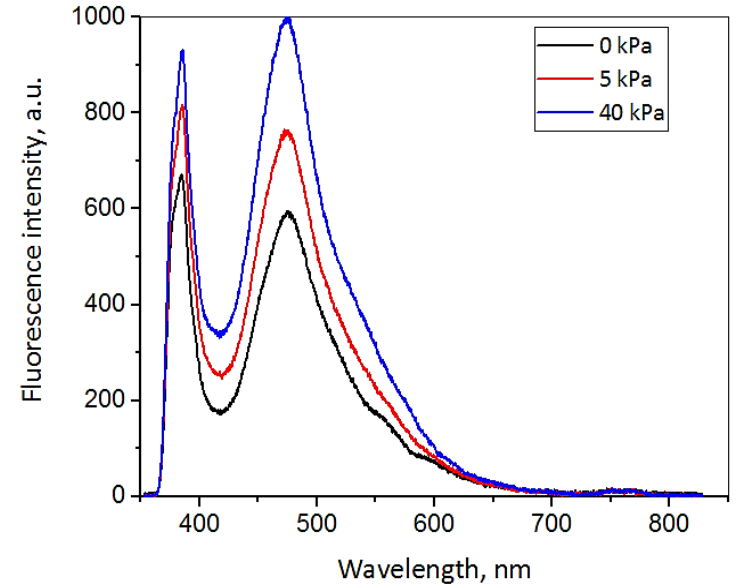

(a)

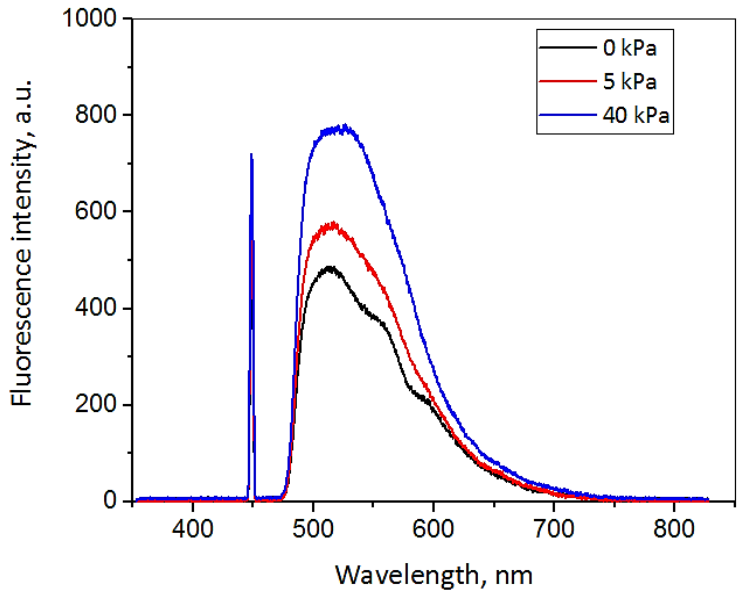

(b)

Figure 4. Typical fluorescence spectra at pressures $0 \mathrm{kPa}, 5 \mathrm{kPa}$ and $40 \mathrm{kPa}$ for wavelengths $365 \mathrm{~nm}$ (a) $450 \mathrm{~nm}$ (b) The obtained experimental data were used to get averaged curves of increasing fluorescence intensity with increasing probe pressure (Fig.5).

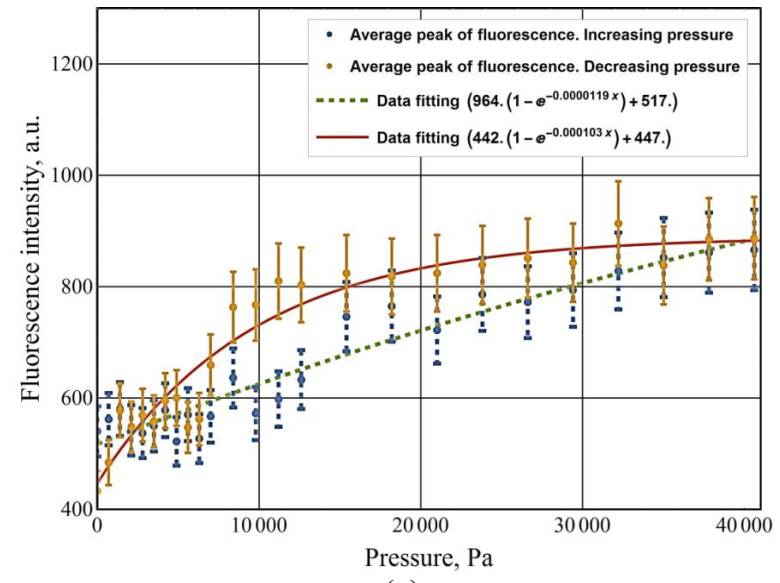

(a)

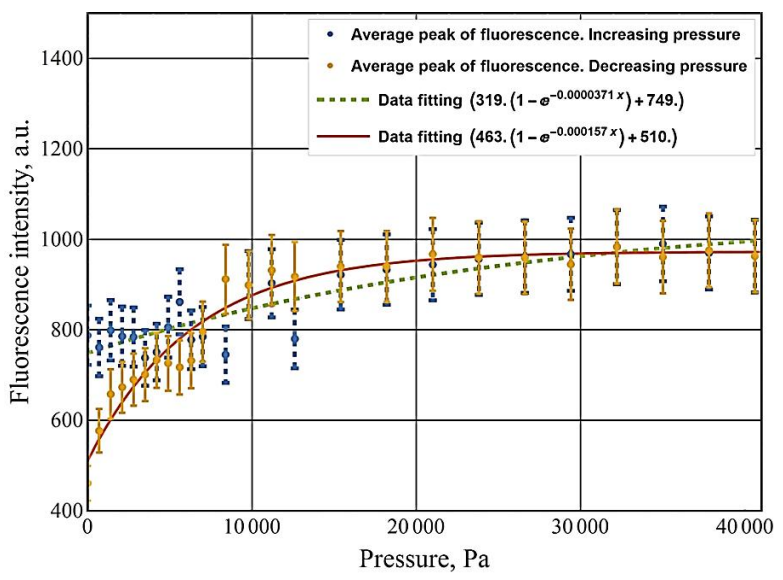

(b)

Figure 5. Average peaks of fluorescence intensity for all volunteers for wavelengths $365 \mathrm{~nm}$ (a) $450 \mathrm{~nm}$ (b)

At pressure $40 \mathrm{kPa}$ fluorescence intensity increased at excitation wavelengths $\lambda=365 \mathrm{~nm}$ by $95 \%, \lambda=450 \mathrm{~nm}$ by $105 \%$ and $\lambda=532 \mathrm{~nm}$ by $40 \%$. Moreover, fluorescence intensity increase at $5 \mathrm{kPa}$ reached $30 \%$ at $\lambda=365 \mathrm{~nm}, 25 \%$ at $\lambda=450$ $\mathrm{nm}, 22 \%$ at $\lambda=532 \mathrm{~nm}$. At excitation wavelength $\lambda=637 \mathrm{~nm}$, no significant influence of pressure on fluorescence intensity was revealed. Registered increase of fluorescence intensity at aforementioned wavelengths can be explained by decreasing blood content in sampling volume due to pressure increase.

\section{CONCLUSION}

Thus, pressure on optical probe has sufficient impact on skin microcirculation to affect registered fluorescence intensity. Data generated in this study are of interest for design and development of diagnostic technologies for wearable devices. This data will also inform further investigation into issues of compensation of blood absorption influence on fluorescence spectrum, allowing increased accuracy and reproducibility of measurements by fluorescence spectroscopy methods in optical diagnosis. 


\section{ACKNOWLEDGMENTS}

The work was supported by grant of the President of the Russian Federation for state support of young Russian scientists № MK-7168.2016.8 as well as partially by Finish Academy, grant №296408.

\section{REFERENCES}

[1] I. Meglinski, D. A. Greenhalgh, S. J. Matcher, "Effect of mechanical pressure on the skin surface produced by fibreoptic probe in a blood microcirculation study," in Biomedical Topical Meeting, Optical Society of America, TuD16, (2002).

[2] I. Meglinski, D. A. Greenhalgh, "Skin blood microcirculation probing: experiments and theoretical remarks," Proc. SPIE 4707, Saratov Fall Meeting 2001: Optical Technologies in Biophysics and Medicine III, 194 (2002)

[3] V. V. Dremin, E. A. Zherebtsov, I. E. Rafailov, A. Y. Vinokurov, I. N. Novikova, A. I. Zherebtsova, K. S. Litvinova, Dunaev A. V., "The development of attenuation compensation models of fluorescence spectroscopy signals," Proc. SPIE 9917, Saratov Fall Meeting 2015: Third International Symposium on Optics and Biophotonics and Seventh FinnishRussian Photonics and Laser Symposium (PALS), 99170Y (2016).

[4] S. L. Jacques, "Optical properties of biological tissues: A review," Physics in Medicine and Biology, 58(11), 37 (2013).

[5] V. Kalchenko, N. Madar-Balakirski, I. Meglinski, A. Harmelin, "In vivo characterization of tumor and tumor vascular network using multi-modal imaging approach," Journal of Biophotonics, 4(9), 645-649 (2011).

[6] V. Kalchenko, Kuznetsov, I. Meglinski, A. Harmelin, "Label free in vivo laser speckle imaging of blood and lymph vessels," J Biomed Opt, 17(5), 050502 (2012).

[7] V. Kalchenko, D. Israeli, Y. Kuznetsov, I. Meglinski, A. Harmelin, "A simple approach for non-invasive transcranial optical vascular imaging (nTOVI)," J Biophotonics, 8, 897-901 (2015).

[8] J. R. Lakowicz, [Principles of Fluorescence Spectroscopy], Kluwer Academic Publishers, 954 (2006).

[9] M. A. Mycek, B. W. Pogue, "Handbook of Biomedical Fluorescence," CRC Press, (2003).

[10] A. V. Dunaev, V. V. Sidorov, A. I. Krupatkin, I. E. Rafailov, S. G. Palmer, N. A. Stewart, S. G. Sokolovski, E. U. Rafailov, "Investigating tissue respiration and skin microhaemocirculation under adaptive changes and the synchronization of blood flow and oxygen saturation rhythms," Physiological Measurement, 35(4), 607-621 (2014).

[11] A. V. Dunaev, V. V. Dremin, E. A. Zherebtsov, I. E. Rafailov, K. S. Litvinova, S. G. Palmer, N. A. Stewart, S. G. Sokolovski, E. U. Rafailov, "Individual variability analysis of fluorescence parameters measured in skin with different levels of nutritive blood flow," Medical Engineering and Physics, 37(6), 574-583 (2015).

[12] M. J. Leahy, F. F. M. De Mul, G. E. Nilsson, R. Maniewski, "Principles and practice of the laser-Doppler perfusion technique," Technology and Health Care, 7(2), 143-162 (1999).

[13] V. V. Tuchin, [Tissue optics: Light scattering methods and instruments for medical diagnosis: Third edition], SPIE PRESS BOOK (2015). 